OPEN ACCESS

Edited by:

Santanu Bose,

Washington State University, USA

Reviewed by:

Michael Teng,

University of South Florida, USA

Ahmed Sabbah,

The University of Texas Health Science Center at San Antonio, USA

*Correspondence:

Qinghua Nie

nqinghua@scau.edu.cn

Received: 12 September 2016 Accepted: 13 January 2017 Published: 30 January 2017

Citation:

Li Z, LUo Q, Xu H, Zheng $M$ Abdalla $B A$, Feng $M$, Cai $B$, Zhang $X$,

Nie $Q$ and Zhang X (2017)

MiR-34b-5p Suppresses Melanoma

Differentiation-Associated Gene 5 (MDA5) Signaling Pathway to Promote

Avian Leukosis Virus Subgroup $J$ (ALV-J)-Infected Cells Proliferaction and ALV-J Replication.

Front. Cell. Infect. Microbiol. 7:17. doi: 10.3389/fcimb.2017.00017

\section{MiR-34b-5p Suppresses Melanoma Differentiation-Associated Gene 5 (MDA5) Signaling Pathway to Promote Avian Leukosis Virus Subgroup J (ALV-J)-Infected Cells Proliferaction and ALV-J Replication}

\author{
Zhenhui Li ${ }^{1,2}$, Qingbin Luo ${ }^{1,2}$, Haiping Xu ${ }^{1,2}$, Ming Zheng ${ }^{1,2}$, Bahareldin Ali Abdalla ${ }^{1,2}$, \\ Min Feng ${ }^{1,2}$, Bolin Cai ${ }^{1,2}$, Xiaocui Zhang ${ }^{1,2}$, Qinghua Nie ${ }^{1,2 *}$ and Xiquan Zhang ${ }^{1,2}$ \\ ${ }^{1}$ Department of Animal Genetics, Breeding and Reproduction, College of Animal Science, South China Agricultural \\ University, Guangzhou, China, ${ }^{2}$ Guangdong Provincial Key Lab of Agro-Animal Genomics and Molecular Breeding and the \\ Key Lab of Chicken Genetics, Breeding and Reproduction, Ministry of Agriculture, Guangzhou, China
}

Avian leukosis virus subgroup $J(A L V-J)$ is an oncogenic retrovirus that has a similar replication cycle to multiple viruses and therefore can be used as a model system for viral entry into host cells. However, there are few reports on the genes or microRNAs (miRNAs) that are responsible for the replication of ALV-J. Our previous miRNA and RNA sequencing data showed that the expression of miR-34b-5p was significantly upregulated in ALV-J-infected chicken spleens compared to non-infected chicken spleens, but melanoma differentiation-associated gene 5 (MDA5) had the opposite expression pattern. In this study, a dual-luciferase reporter assay showed that MDA5 is a direct target of miR-34b-5p. In vitro, overexpression of miR-34b-5p accelerated the proliferation of ALV-J-infected cells by inducing the progression from G2 to S phase and it promoted cell migration. Ectopic expression of MDA5 inhibited ALV-J-infected cell proliferation, the cell cycle and cell migration, and knockdown of MDA5 promoted proliferation, the cell cycle and migration. In addition, during ALV-J infections, MDA5 can detect virus invasion and it triggers the MDA5 signaling pathway. MDA5 overexpression can activate the MDA5 signaling pathway, and thus it can inhibit the mRNA and protein expression of the ALV-J env gene and it can suppress virion secretion. In contrast, in response to the knockdown of MDA5 by small interfering RNA (siRNA) or an miR-34b-5p mimic, genes in the MDA5 signaling pathway were significantly downregulated $(P<0.05)$, but the mRNA and protein expression of ALV-J env and the sample-to-positive ratio of virion in the supernatants were increased. This indicates that miR-34b-5p is able to trigger the MDA5 signaling pathway and affect ALV-J infections. Together, these results suggest that miR-34b-5p targets MDA5 to accelerate the proliferation and migration of ALV-J-infected cells, and it promotes ALV-J replication, via the MDA5 signaling pathway.

Keywords: MiR-34b-5p, melanoma differentiation-associated gene 5 (MDA5), Avian leukosis virus subgroup $J$ (ALV-J), Cell proliferation, MDA5 signaling pathway 


\section{INTRODUCTION}

Avian leukosis virus subgroup J (ALV-J) is a novel, virulent subtype in the avian leukosis virus family (ALVs), which can be classified into 10 subgroups (Payne, 1998). In birds, ALV-J can induce myeloid leukosis, various tumors, growth retardation, and serious immunosuppression (Stedman and Brown, 1999; Payne and Nair, 2012; Zeng et al., 2014). There is no effective vaccine against ALV-J (Payne and Nair, 2012). Furthermore, as an oncogenic retrovirus, ALV-J shares a similar replication cycle with a number of RNA and DNA viruses, such as human immunodeficiency virus type-1 (HIV-1), which can replicate in both the nucleus and the cytoplasm. Therefore, it can be used as a model system for viral entry into host cells. Thus, identifying the pathogenic mechanism of ALV-J can provide a solid foundation for further study of other retroviruses. Recently, several genes and the extracellular signal-regulated kinase $(\mathrm{ERK}) /$ mitogen-activated protein kinase (MAPK) pathway have been demonstrated to be important for ALV-J replication and oncogenesis (Chai and Bates, 2006; Li Y. et al., 2014; Dai et al., 2016). However, the microRNAs (miRNAs) and pathways involved in ALV-J infections largely remain unclear.

MiRNAs are small non-coding RNAs that play important roles in cell growth, proliferation, and apoptosis by inducing translational inhibition or mRNA degradation (Sun et al., 2010; Feng et al., 2011). Aberrant expression of miRNAs has been linked to tumorigenesis (Zhao et al., 2009; Datta et al., 2012). To date, the expression of several miRNAs has been found to change in response to ALV-J infection. For example, four miRNAs (ggamiR-125b, gga-miR-193a, gga-miR-193b, and gga-miR-221) that are known to be involved in tumorigenesis-related pathways were shown to be expressed abnormally in ALV-J-infected chickens, and they might be associated with ALV-J-induced tumorigenesis (Wang et al., 2013a). It has been shown that the binding of ggamiR-1650 to several sites in the $5^{\prime}$ untranslated region (UTR) of ALV-J genomic RNA is critical for viral replication (Wang et al., 2013b). In addition, gga-miR-221, gga-miR-222, and gga-miR375 have been shown to play a pivotal role in tumorigenesis after an ALV-J infection (Li H. et al., 2014; Dai et al., 2015).

We previously showed that miRNA-23b promotes ALV-J replication by targeting interferon regulatory factor-1 (IRF1; Li et al., 2015). The miRNA sequencing data (Gene Expression Omnibus; GEO database, accession number GSE63676) also showed that another miRNA, gga-miR-34b-5p, was upregulated in ALV-J-infected chickens compared to non-infected chickens, suggesting that this miRNA might also be involved in ALV-J infections. In humans, miR-34b has been reported to be involved in many neoplastic diseases and low levels of expression of miR$34 \mathrm{~b}$ were found to be associated with gastric tumorigenesis (Tsai et al., 2011).

Vertebrate innate immune responses are mediated by pattern recognition receptors (PRRs), including Toll-like receptors (TLRs), retinoic acid-inducible gene I-like receptors (RLRs), and Nod-like receptors (NLRs). MDA5 is a member of the RLR family, which are comprised of $\mathrm{N}$-terminal tandem caspase activation and recruitment domains (CARDs), a central helicase domain responsible for RNA-dependent ATP hydrolysis and a C-terminal regulatory domain (CTD; Kato et al., 2006). Moreover, MDA5 can activate the interferon (IFN) signaling pathway and it thereby plays a critical role in antiviral innate immunity. Our previously published RNA sequencing data (GSE63226) showed that MDA5 was downregulated in ALV-Jinfected chickens compared to non-infected chickens. However, relatively little is known regarding the effect of ectopic expression of MDA5 in ALV-J-infected chickens.

The first aim of this study was to explore whether or not miR$34 \mathrm{~b}-5 \mathrm{p}$ is involved in ALV-J infections and to clarify how it affects ALV-J replication, as well as to characterize the oncogenesis in chicken fibroblast cell line (DF-1) infected with ALV-J. We confirmed that miR-34b-5p was increased in ALV-J-infected tissues and that ectopic expression of miR-34b-5p accelerated the proliferation and migration of ALV-J-infected cells. MDA5 was verified as a direct target of miR-34b-5p. Further in vitro investigations demonstrated that miR-34b-5p can regulate the MDA5 signaling pathway, the expression of the ALV-J Env gene, and virion secretion. Taken together, these results suggest that miR-34b-5p accelerates the proliferation and migration ALVJ-infected cells and promotes ALV-J replication by targeting MDA5.

\section{MATERIALS AND METHODS Ethics Statement}

All animal experiments in this study were performed following protocols approved by the Institutional Animal Care and Use Committee at the South China Agricultural University, China (approval ID: SCAU\#0011). All the experiments were carried out in compliance with the regulations and guidelines established by this committee. The authors declare that all animal experiments under the consent of the animal owners. All efforts were made to minimize suffering to animal. The authors declare no additional considerations of the study in cases where vulnerable populations were involved.

\section{Animals}

Two chicken groups, ALV-J-infected broilers and non-infected broilers at 140 days old, were used. The animals were the same as those used in our previous RNA sequencing study (Li et al., 2015).

\section{Virus Propagation and Quantification of Virus Titer}

SCAU-HN06, a strain of ALV-J, was kindly provided by Assoc. Prof. Weisheng Cao (South China Agricultural University). The chicken embryonic fibroblast cell line (DF-1 cells) was obtained from the Cell Bank of the Committee on Type Culture Collection of the Chinese Academy of Sciences and it was cultivated adherently in cell culture vessels containing Dulbecco's Modified Eagle's Medium (DMEM; Gibco, Life Technologies, Grand Island, NY, USA) supplemented with 10\% fetal bovine serum (FBS; Gibco, Life Technologies, Grand Island, NY, USA) at $37^{\circ} \mathrm{C}$ in an atmosphere of $5 \% \mathrm{CO}_{2}$. When the cells grew to a density of $80 \%$ confluence, they were infected with the ALV-J strain SCAU-HN06. The inoculum was discarded $2 \mathrm{~h}$ 
later and it was replaced with DMEM containing 1\% FBS, 100 $\mathrm{U} / \mathrm{mL}$ penicillin, and $100 \mathrm{ug} / \mathrm{mL}$ streptomycin. After incubation for 6 days in culture, the cell supernatants (which contained the viral particles) were harvested as described previously (Dai et al., 2016). The virus was diluted 10 -fold using serial dilution techniques in DMEM and the suspension was then applied to DF-1 cells for 6 days in a 96-well plate. The virus was titrated to determine the $50 \%$ tissue culture infectious doses $\left(\right.$ TCID $\left._{50}\right)$ in accordance with the method described by Reed and Muench (1938).

\section{RNA Isolation and cDNA Synthesis}

Total RNA was extracted from the tissue samples and DF-1 cells using TRIzol ${ }^{\circledR}$ as recommended by the supplier (Invitrogen Life Technologies, Carlsbad, CA, USA). The integrity and quantity of RNA were assessed using 1.5\% agarose gel electrophoresis and spectrophotometry (ND-2000, NanoDrop Inc., Wilmington, DE, USA), respectively. A PrimeScript RT Reagent Kit (Takara, Otsu, Japan) was used to carry out cDNA synthesis of mRNA, following the manufacture's protocol. The synthesized cDNA was stored at $-20^{\circ} \mathrm{C}$ until subsequent analysis using real-time quantitative polymerase chain reaction (RT-qPCR).

\section{Primers}

RT-qPCR primers that were specific for MDA5, ALV-J Env, Gag, Pol, $M x 1$, and $\beta$-actin were designed using Premier Primer 5.0 software. RT-qPCR primers that were specific for genes in the MDA5 signaling pathway, including interferon- $\beta$ promoter stimulator 1 (IPS-1), interferon regulatory factor-3 (IRF-3), interferon- $\beta$ (IFN $\beta)$, double-stranded RNA-dependent protein kinase $(P K R), 2^{\prime}, 5^{\prime}$-oligoadenylate synthetase $(O A S)$, myxovirus (influenza virus) resistance 1 , interferon-inducible protein $\mathrm{p} 78$ (mouse) (Mx1), and major histocompatibility complex class I (MHC class I), are referenced in previous publications (Supplementary Table 1; Lee C. C. et al., 2014). PCR primers for the full-length MDA5 coding sequence clone and the $3^{\prime}$ UTR of the MDA5 clone were also designed using the Premier Primer 5.0 software (Supplementary Table 2). All the above primers were synthesized by Sangon Biotech Co., Ltd. (Guangzhou, China). A bulge-loop ${ }^{\mathrm{TM}}$ Reverse Transcription primer and RT-qPCR primers that were specific for gga-miR-34b-5p were designed and synthesized by RiboBio (Guangzhou, China).

\section{RNA Oligoribonucleotides and Plasmids Construction}

Gga-miR-34b-5p mimics, mimic control duplexes, small interfering RNA (siRNA) targeted against the MDA5 gene (si-MDA5), and a non-specific siRNA control duplex were designed and synthesized by RiboBio (Guangzhou, China). Oligonucleotide sequences of si-MDA5 were showed in Supplementary Table 3. To construct a gga-miR-34b-5p target luciferase reporter vector (pmirGLO-WT MDA5-3'UTR), the segment sequence of the MDA5 $3^{\prime}$ UTR (666 bp) that contained the putative gga-miR-34b-5p binding sequence was amplified by PCR using a cDNA template synthesized from total RNA. Subsequently, the PCR product was sub-cloned into NheI/SalI restriction sites in the pmirGLO dual-luciferase reporter vector
(Promega, Madison, WI, USA) to generate the pmirGLOWT-MDA5-3'UTR reporter vector.

However, to generate a gga-miR-34b-5p target-mutated reporter vector (pmirGLO-MT-MDA5-3'UTR), mutations were achieved by changing the gga-miR-34b-5p binding seed sequences from ACTGCCT to GACTATC using the megaprimer PCR method (Ke and Madison, 1997). An MDA5 overexpression construct was generated by amplifying the MDA5 coding sequence, and it was subsequently cloned into the overexpression plasmid vector, pSDS-20218, which was purchased from Shanghai SiDanSai Biotechnology Co., Ltd., China (http://www. sidansai.com/).

\section{Transfection of MDA5 Overexpression Plasmid, si-MDA5, and miR-34b-5p Mimics and Preparation of ALV-J}

When DF-1 cells grew to a density of $50 \%$ confluence, they were transfected with (a) the MDA5 overexpression plasmid, (b) siMDA5, or (c) the gga-miR-34b-5p mimic using Lipofectamine 3000 Reagent (Life Technologies, USA), in accordance with the manufacture's recommended protocol., After $12 \mathrm{~h}$, the cells were inoculated with $\mathrm{TCID}_{50}$ of ALV-J. After $2 \mathrm{~h}$ of incubation, the supernatant was discarded and the infected cells were replenished with DMEM medium containing $1 \%$ FBS, $100 \mathrm{U} / \mathrm{mL}$ penicillin, and $100 \mathrm{ug} / \mathrm{mL}$ streptomycin.

\section{Luciferase Reporter Assay}

Luciferase activity was measured using Dual-GLO ${ }^{\circledR}$ Luciferase Assay System Kits (Promega, Madison, WI, USA) following the manufacturer's instructions. DF-1 cells were seeded at a density of $1 \times 10^{3}$ cells per well in 96-well plates. After $24 \mathrm{~h}$, the cells were co-transfected with $100 \mathrm{ng}$ pmir-GLOWT-MDA5-3' UTR- (wild-type) or pmir-GLO-MT-MDA5-3' UTR(mutant-type) plasmids, or $100 \mathrm{nM}$ gga-miR-34b-5p mimic and miR-NC using the Lipofectamine 3000 Reagent. Fourty-eight hours after transfection, luciferase assays were performed using a Fluorescence/Multi-Detection Microplate Reader (Synergy 2, Biotek, Winooski, VT, USA). The values obtained were normalized to the levels of a Renilla luciferase plasmid (pRL-TK Vector) levels.

\section{RT-qPCR Analysis}

The RT-qPCR reactions were performed using a Bio-Rad CFX96 Real-Time PCR Detection System using iTaq ${ }^{\text {TM }}$ Universal SYBR $^{\circledR}$ Green Supermix Kits (Bio-Rad Laboratories Inc., Hercules, CA, USA) to detect the mRNA expression level associated with each gene. The chicken $\beta$-actin gene was used as an internal control. The analysis was carried out using the $2^{-\Delta \Delta \mathrm{Ct}}$ method, as described previously (Livak and Schmittgen, 2001).

\section{Wound Healing Assay}

We also carried out a wound healing migration assay. First, DF-1 cells were seeded in 12-well plates. When the cells grew to a density of $50 \%$ confluence, they were transfected with the MDA5 overexpression plasmid, si-MDA5, or a gga-miR-34b-5p mimic, and then they were infected with ALV-J, as described 
above. When the cells reached a density of $100 \%$ confluence, a linear wound was generated by scratching the monolayer of cells with a $1 \mathrm{~mL}$ pipette tip. The cells that migrated into the wound area were captured at 0 (control), 24, 48, and $72 \mathrm{~h}$ using a microscope (Nikon, Shinagawa, Tokyo, Japan). Width of the scratches was measured using the Image Pro Plus 6.0 software (Media Cybernetics). The data of each scratch width were presented in Supplementary Table 4 . The formula to calculate the cell migration rate was as follows: $\left(\mathrm{W}_{0 \mathrm{~h}}-\mathrm{W}_{\mathrm{xh}}\right) \times 100 \% / \mathrm{W}_{0 \mathrm{~h}}$, where $\mathrm{W}_{0 \mathrm{~h}}$, represents the mean wound width at $0 \mathrm{~h}$ and $\mathrm{W}_{\mathrm{xh}}$ represents the mean wound width at 24,48 , and $72 \mathrm{~h}$.

\section{Flow Cytometry Analysis}

For the flow cytometry analysis of the cell cycle, DF-1 cells were seeded in 12-well plates. When the cells grew to a density of $50 \%$ confluence, they were transfected and then infected with ALV-J, as described above. After being infected, the cells were incubated for $72 \mathrm{~h}$, collected and fixed overnight with $75 \%$ ethanol at $4^{\circ} \mathrm{C}$. The fixed cells were stained with $50 \mu \mathrm{g} / \mathrm{mL}$ propidium iodide solution (Sigma Life Science, St. Louis, MO, USA) containing $10 \mu \mathrm{g} / \mathrm{mL}$ RNase A (Takare) and $0.2 \%(\mathrm{v} / \mathrm{v})$ Triton X-100 (Sigma), and they were incubated in the dark for $30 \mathrm{~min}$ at $37^{\circ} \mathrm{C}$. The cell cycle phase analysis of the incubated cells was performed using a BD Accuri C6 flow cytometer (BD, San Jose, CA, USA).

\section{Sodium Dodecyl Sulfate Polyacrylamide Gel Electrophoresis (SDS-PAGE) and Western Blotting}

SDS-PAGE and Western blotting were performed using methods that have been described in detail previously (Feng et al., 2016). The primary antibodies used were as follows: ALV-J-specific monoclonal antibody JE-9 (which was kindly provided by Prof. Aijian Qin, Yangzhou University, China) and mouse polyclonal anti-glyceraldehyde 3-phosphate dehydrogenase (anti-GAPDH; Bioworld Technology, Inc., USA). Goat anti-rabbit IgG (heavy and light chains $(\mathrm{H}+\mathrm{L})$ )-horseradish peroxidase (HRP) and goat anti-mouse IgG $(\mathrm{H}+\mathrm{L})$-HRP (Bioworld Technology, Inc., USA) served as secondary antibodies. The DF-1 cells were seeded in 6well plates. When the cells grew to a density of $50 \%$ confluence, they were transfected, and then infected with ALV-J, as described above. After 3 days in culture, the DF-1 cells were subjected to the SDS-PAGE and Western blot analyses. Detection of HRPconjugated secondary antibodies was performed using Western Lightning Chemiluminescence Reagent Plus (Perkin Elmer Life Sciences, Inc, Boston, MA, USA), and images were developed on $\mathrm{X}$-ray films.

\section{Enzyme-Linked Immunosorbent Assay (ELISA)}

For the ELISA, the DF-1 cells were seeded in 96-well plates. When the cells grew to a density of $50 \%$ confluence, they were transfected, and then infected with ALV-J, as described above. At the indicated times $(24,48,72,96$, and $120 \mathrm{~h})$, the supernatants were collected. After three repetitions of freezing and thawing the supernatants, the ALV-J p27 protein expression level was measured using an ELISA ALV-J diagnosis kit, as described in the manufacturer's protocol (IDEXX, USA).

\section{Cell-Counting Kit-8 (CCK-8) Assay}

For the cell growth assays, DF-1 cells were seeded in 96-well plates. When the cells grew to a density of $50 \%$ confluence, they were transfected, and then infected with ALV-J, as described above. The viable cells were detected every $24 \mathrm{~h}$ using a CKK-8 (Dojindo, Kumamoto, Japan), in accordance with the manufacturer's protocol. Absorbance was measured using a Model 680 Microplate Reader (Bio-Rad, Hercules, California, USA) by optical density at a wavelength of $450 \mathrm{~nm}$.

\section{Statistical Analysis}

Each experiment was performed in triplicate. The data are presented as the mean \pm the standard error of the mean (SEM) of each set of three independent experiments. Where applicable, the statistical significance of the data was tested using one-sample or paired $t$-tests. The types of tests and the $P$-values, when applicable, are indicated in the figure legends.

\section{RESULTS}

\section{MIR-34b-5p is Aberrantly Upregulated in ALV-J-Infected Chickens}

ALV-J, an oncogenic retrovirus, induces several leukemia-like proliferative diseases in the hemopoietic systems of chickens. Based on our previous miRNA sequencing data (GEO database accession numbers, GSE63676), miR-34b-5p was found to be significantly $(P=1.62 \mathrm{E}-71)$ upregulated in the spleens of ALVJ-infected chickens compared to the spleens of non-infected chickens (Figure 1A). In addition, RT-qPCR involving immune system-related tissues showed that the expression of miR-34b$5 \mathrm{p}$ was significantly increased $(P=0.0036$ and $P=0.0197)$ in ALV-J-infected spleens and thymuses compared to non-infected spleens and thymuses, respectively (Figure 1B). In the liver, the expression of miR-34b-5p was also increased in ALV-J-infected chickens, although the difference was not significant $(P=0.09)$. These findings demonstrate that the expression of miR-34b-5p is significantly dysregulated in ALV-J-infected chickens and it might be involved in virus invasion.

\section{MIR-34b-5p Promotes Cell Proliferation and Migration and Modulates Cell Cycle Progression in ALV-J-Infected DF-1 Cells}

Previous studies demonstrated that miRNAs can affect various aspects of cancer biology, including cell proliferation, migration, and apoptosis (Zhao et al., 2014; Shi W. et al., 2016). To explore the role of miR-34b-5p on ALV-J infection, we investigated its influence on cellular processes in ALV-J-infected cells. Firstly, we transiently transfected an miR-34b-5p mimic or a negative control mimic (empty plasmid; miR-NC) into DF-1 cells. After $12 \mathrm{~h}$ in culture media, the transfected DF-1 cells were infected with ALV-J, and then cell proliferation and migration analyses were carried out. The result of the CCK- 8 assay showed that overexpression of miR-34b-5p could significantly (at $P<0.05$ or $P<0.01)$ promote the proliferation of ALV-J-infected 


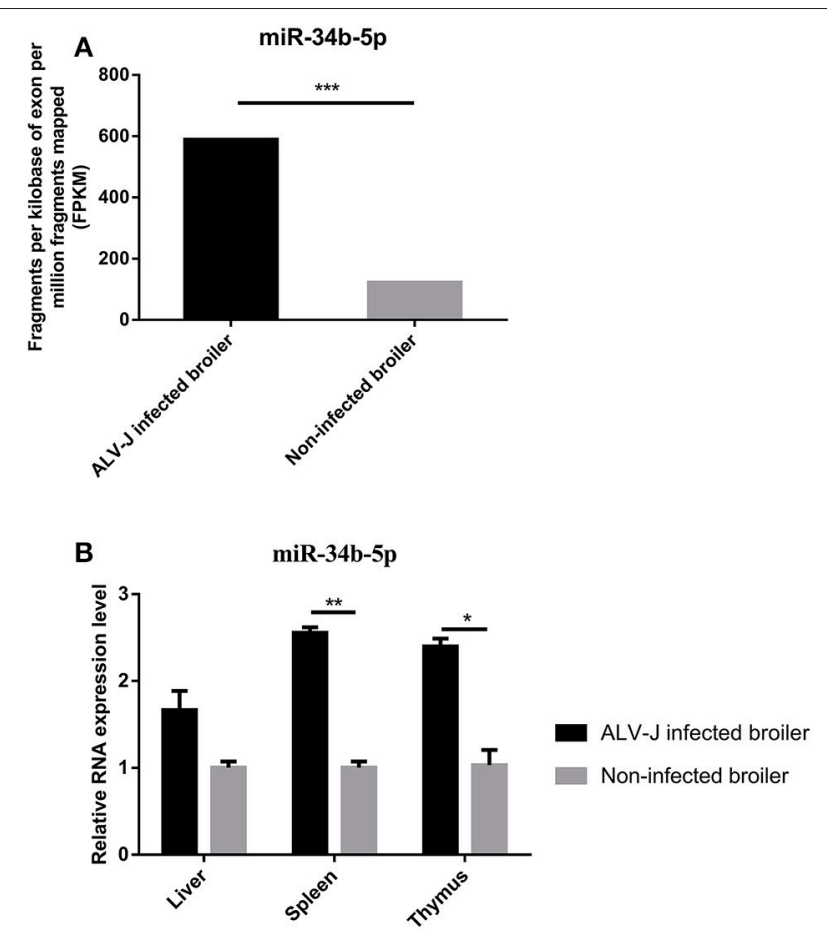

FIGURE 1 | Relative expression of miR-34b-5p in ALV-J-infected and non-infected tissues. (A) MiRNA sequencing showed that miR-34b-5p was upregulated in the spleen of ALV-J-infected chickens. (B) RT-qPCR showed that the expression of miR-34b-5p was increased in immune system-related tissues (including the liver, spleen, and thymus) from ALV-J-infected chickens compared to those from non-infected chickens. RT-qPCR data are expressed as the fold change relative to the control ( $\beta$-actin) gene. In both panels, each bar represents the mean \pm the SEM of three independent experiments. Asterisks denote statistically significant differences: ${ }^{*}$, ${ }^{* *}$, and ${ }^{* * *}$ indicate $P<$ $0.05, P<0.01$, and $P<0.001$, respectively. ALV-J, Avian leukosis virus subgroup J; miRNA, microRNA; RT-qPCR, real-time quantitative polymerase chain reaction; SEM, standard error of the mean.

cells compared to miR-34b-5p negative control (miR-NC) at 24 96 h after the ALV-J infection (Figure 2A). Moreover, using flow cytometry, we found that overexpression of miR-34b-5p significantly increased the number of cells in $S$ phase $(P<0.001)$ and reduced the number of cells in G2 phase $(P<0.05)$ compared to the negative control group (Figure 2B).

Subsequently, to assess the effect of miR-34b-5p overexpression on the cell migration, a wound healing assay was performed in ALV-J-infected DF-1 cells. We found that the migration rate of DF-1 cells transfected with miR-34b-5p mimics was significantly increased $(P<0.05$ and $P<0.01$ at 48 and $72 \mathrm{~h}$ after the ALV-J infection, respectively) compared to the negative control group (Figures 2C,D). Taken together, our data suggested that miR-34b-5p promotes the growth of ALV-J-infected DF-1 cells by accelerating their proliferation and migration.

\section{MDA5 is a Direct Target of miR-34b-5p}

Our previous RNA sequencing data (GSE63226) demonstrated that MDA5 was significantly downregulated $(P=1.34 \mathrm{E}-06)$ in the spleens of ALV-J-infected chickens compared to noninfected chickens (Figure 3A). The RT-qPCR results revealed that the expression of MDA5 was significantly downregulated in immune system-related tissues including the livers $(P=$ $0.046)$, spleens $(P=0.0022)$, and thymuses $(P=0.022)$ of ALV-J-infected chickens compared to those from noninfected chickens (Figure 3B). In addition, miR-34b-5p had a contrasting expression pattern in ALV-J-infected chickens compared to non-infected chickens (Figures 1, 3A,B). Using miRNA target prediction software, miRDB (http://www.mirdb. org/miRDB) (Wong and Wang, 2015), MDA5 was predicted to be a potential target gene for miR-34b-5p. The miR-34b-5p mature sequence and its binding seed sequence in MDA5 are shown in Figure 3C. To confirm whether miR-34b-5p directly targets the $3^{\prime}$ UTR of MDA5, a dual-luciferase reporter gene assay was carried out in DF-1 cells. The transfection efficiency analysis showed that 50 and $100 \mathrm{nM}$ of an miR-34b-5p mimic could significantly $(P=0.019)$ and highly significantly $(P=$ $0.0027)$ decrease the MDA5 expression levels in DF-1 cells at $48 \mathrm{~h}$ after transfection, respectively (Figure 3D). Therefore, we co-transfected $100 \mathrm{nM}$ miR-34b-5p mimic or mi-NC into DF-1 cells with reporter vectors. The dual luciferase assay showed that the wild-type group (WT-MDA5-3'UTR) induced a significant decrease $(P<0.05)$ in luciferase activity compared to the control group, whereas no significant difference was observed between the mutant-type group (MT-MDA5-3'UTR) and control group (Figure 3E). Taken together, these results suggested that MDA5 is a direct target of miR-34b-5p.

\section{MDA5 Mediates the Effect of miR-34b-5p on Cell Proliferation and Migration}

To explore whether the effect of miR-34b-5p on cell processes in ALV-J-infected cells was mediated by MDA5, we performed CCK-8, cell cycle and wound healing assays in MDA5 overexpression and knockdown DF-1 cells. Overexpression of MDA5 significantly inhibited $(P \leq 0.01)$ cell proliferation compared to the control group at $24-120 \mathrm{~h}$ after the ALV-J infection (Figure 4A). However, MDA5 knockdown significantly promoted $(P \leq 0.05)$ cell proliferation at $48-120 \mathrm{~h}$ after the ALV-J infection (Figure 4B). On the other hand, MDA5 overexpression significantly $(P<0.001)$ decreased the proportion of ALV-Jinfected cells at $S$ phase but notably increased the proportion of cells at G1 phase $(P<0.0001)$ and G2 phase $(P<0.05)$ compared to the control group (Figure 4C). In contrast, knockdown of MDA5 using siRNA led to a significant $(P<0.01)$ elevation in the proportion of cells at $S$ phase, and a clear reduction in the proportion of cells at G1 phase $(P<0.05)$ or $\mathrm{G} 2$ phase $(P$ $<0.01$; Figure 4D). Moreover, the migration rate of DF-1 cells that overexpressed MDA5 was significantly decreased $(P \leq 0.01)$ compared to that in the control group (Figures $4 \mathrm{E}, \mathrm{F}$ ). In contrast, the migration rate of MDA5 knockdown cells was significantly increased $(P \leq 0.01)$ compared to those transfected with the small interfering RNA negative control (si-NC; Figures 4G,H). These results indicated that miR-34b-5p might modulate the proliferation, migration, and cell cycle of ALV-J-infected DF-1 cells by targeting MDA5. 
A

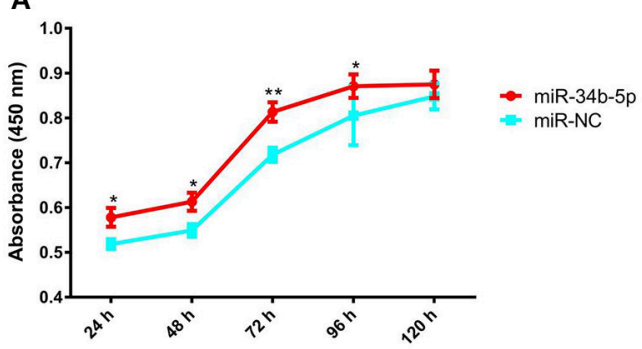

C
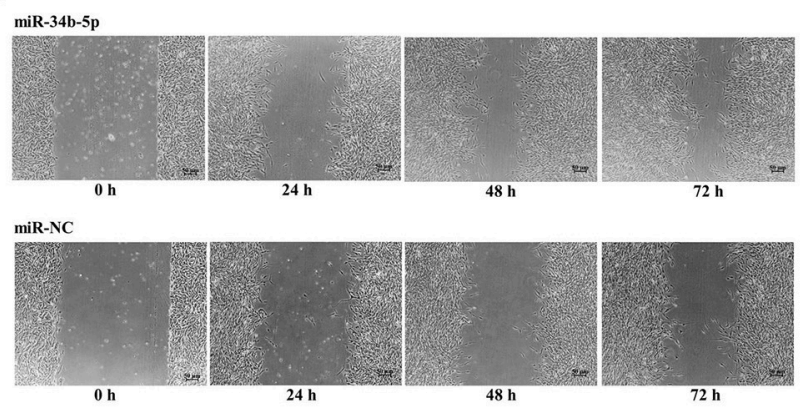

B

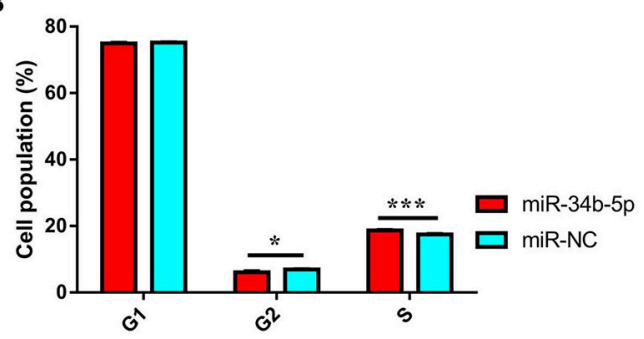

D

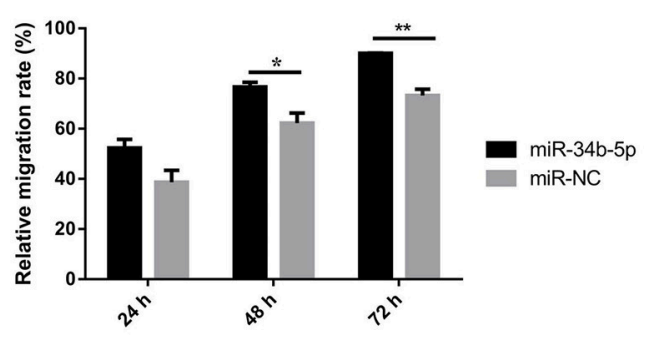

FIGURE 2 | Overexpression of miR-34b-5p promotes the proliferation and migration of ALV-J-infected DF-1 cells and it accelerates G1 to S phase transition. Twelve hours after transfected with miR-34b-5p mimic or mi-NC, the transfected DF-1 cells were infected with ALV-J at TCID 50 . (A) Cell growth was significantly increased after miR-34b-5p mimic transfection compared to the control cells $(n=6)$. (B) Overexpression of miR-34b-5p resulted in an increased proportion of cells in the S phase and a decreased proportion of cells in the G2 phase. (C) Representative images of the wound healing assay at $0,24,48$, and $72 \mathrm{~h}$ after ALV-J infection in miR-34b-5p mimic or mi-NC transfected DF-1 cell. (D) Quantification of wound healing assay at 24, 48, and 72 h after ALV-J infection. In panel $\mathbf{A}$, the results were confirmed by three independent experiments with six (cell number) samples per treatment. In panels (B-D), each bar represents the mean \pm the SEM of three independent experiments. Asterisks denote statistically significant differences: ${ }^{*}$, ${ }^{* *}$, and ${ }^{* \star *}$ indicate $P<0.05, P<0.01$, and $P<0.001$, respectively. ALV-J, Avian leukosis virus subgroup J; mi-NC, mimic-negative control (empty plasmid); UTR, untranslated region; SEM, standard error of the mean; TCID $50,50 \%$ tissue culture infectious dose.

\section{MDA5 Can Detect ALV-J Infections and Trigger the MDA5 Signaling Pathway}

The ALV RNA genome has a typical slowly-transforming replication-competent structure: 5' R-U5-gag-pol-env-U3-R $3^{\prime}$ that contains three coding genes: Gag, Pol, and Env. The env gene codes for an envelope glycoprotein that determines subgroup specificity, neutralization, and receptor binding (Pan et al., 2012). When miR-34b-5p was normal expression (Figure 5A), the expression level of MDA5 was upregulated (Figure 5B) at 16, 24 , and $48 \mathrm{~h}$ after infection compared to at $2 \mathrm{~h}$ after infection. In DF-1 cells infected with ALV-J, we found that the expression of the Env, Gag and Pol mRNA was significantly upregulated at 16,24 , and $48 \mathrm{~h}$ after infection compared to at $2 \mathrm{~h}$ after infection $(P<0.05$; Figures 5C-E). To investigate whether chicken MDA5 signaling pathway was able to detect ALV-J infections and then to initiate and amplify an innate immune response, $\mathrm{RT}-\mathrm{qPCR}$ was used to measure the changes in the expression of innate and antiviral genes in the MDA5 signaling pathway during ALV-J infections. The results showed that the levels of all the mRNA, including those associated with IPS-1, $I R F-3, I F N \beta, P K R, O A S, M x 1$, and $M H C$ class $I$ were upregulated at 24 and $48 \mathrm{~h}$ after infection compared to at $2 \mathrm{~h}$ after infection (Figures 5F-L). In contrast, after overexpression of miR-34b5 p by miRNA mimic (Supplementary Figure 1A) and following inoculation with TCID $_{50}$ of ALV-J, the expression level of MDA5 was downregulated (Supplementary Figure 1B) in miR-34b$5 \mathrm{p}$ overexpression DF-1 cells compared with negative control (NC) group at 2-48 h ALV-J infection time points. In addition, the mRNA expression of ALV-J related genes, including Env, Gag and Pol (Supplementary Figures 1C-E, respectively) were upregulated in miR-34b-5p overexpression DF-1 cells compared with NC group during at 2-48 h ALV-J infection time points. Moreover, the mRNA expression of MDA5 signaling pathwayrelated innate and antiviral genes (IPS-1,IRF-3, IFNB, PKR, OAS, MX1, MHC class 1) were downregulated compared with control (Supplementary Figures 1F-L). These findings indicate that miR34b-5p suppresses MDA5 signaling pathway to promote ALV-J replication.

To further elucidate the function of MDA5 on the MDA5 signaling pathway and ALV-J replication, ectopic expression, and knockdown of MDA5 was carried out in DF-1 cells. In DF-1 cells that overexpressed MDA5, the mRNA and protein expression of Env and the ALV-J virion secretion were significantly suppressed $(P \leq 0.05)$ and the mRNA expression of genes in the MDA5 signaling pathway was significantly upregulated $(P$ $\leq 0.05$; Figures $6 \mathrm{~A}-\mathrm{D})$. In contrast, after knockdown of MDA5 by siRNA, the mRNA and protein expression of env were upregulated $(P<0.05$; Figures 6E,F). The expression of genes 

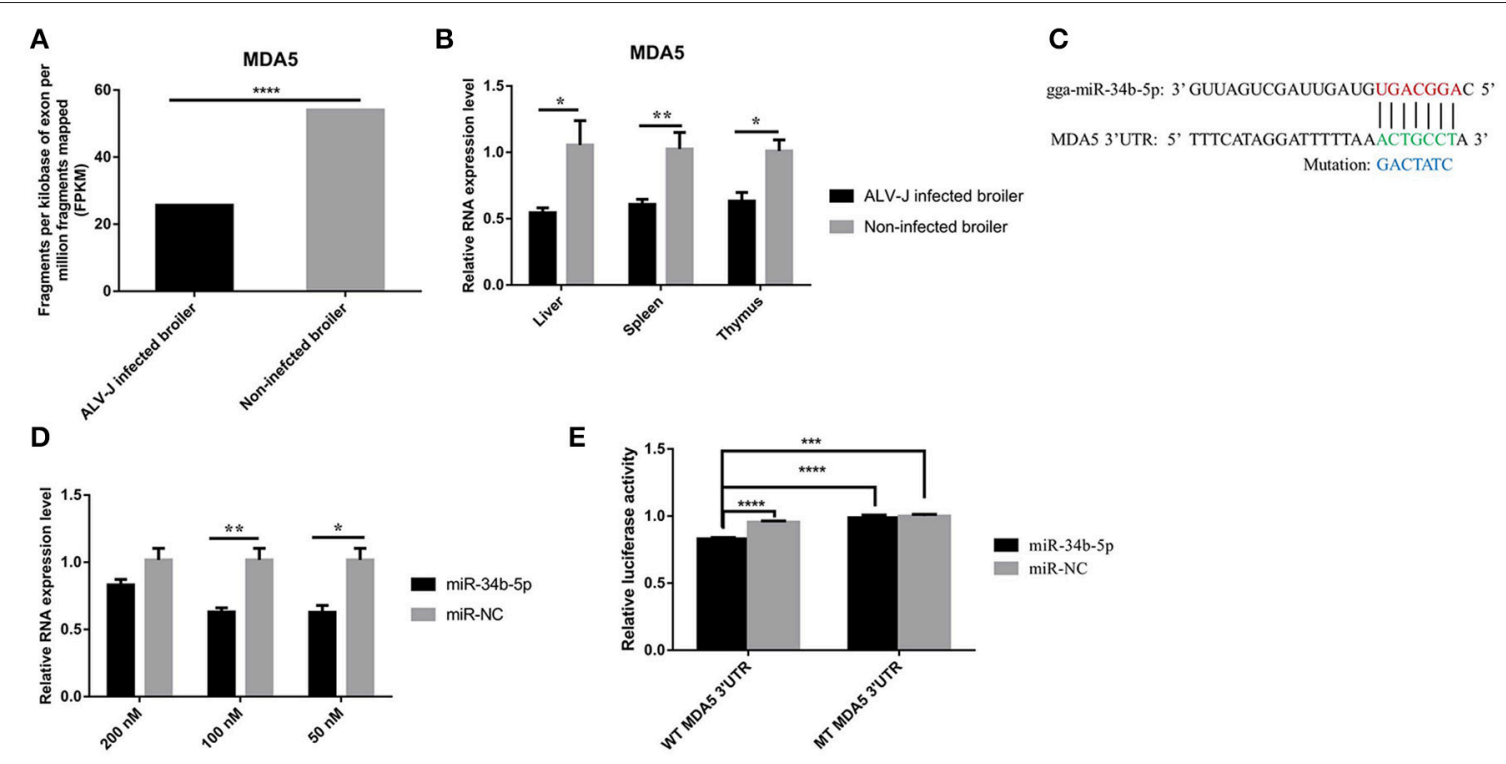

FIGURE 3 | MDA5 is a direct target of miR-34b-5p. (A) RNA sequencing showed that the expression of MDA5 was downregulated in the spleens of ALV-J-infected chickens. (B) RT-qPCR showed that the expression of MDA5 was decreased in immune system-related tissues (including the liver, spleen, and thymus) from ALV-J-infected chickens compared to those from non-infected chickens. (C) MiR-34b-5p binding site in the $3^{\prime}$ UTR of chicken MDA5 mRNA (in green). The mutation sequence in the miR-34b-5p binding site is highlighted in blue. (D) 100 and $50 \mathrm{nM}$ miR-34b-5p mimic could significantly decrease the expression of $M D A 5$ mRNA in DF-1 cells at $48 \mathrm{~h}$ after transfection. (E) The luciferase assay was conducted by cotransfecting wildtype or mutant MDA5 $3^{\prime}$ UTR with $100 \mathrm{nM}$ miR-34b-5p mimic or mi-NC into DF-1 cells. Firefly luciferase activity for each sample was normalized to Renilla luciferase activity. In all panels, the data shown are the mean \pm the SEM of three independent experiments. Asterisks denote statistically significant differences: ${ }^{*},{ }^{* *},{ }^{\star * *}$, and ${ }^{* \star *}$ indicate $P<0.05, P<0.01, P<0.001$, and $P<0.0001$, respectively. ALV-J, Avian leukosis virus subgroup J; MDA5, melanoma differentiation-associated gene 5; mi-NC, mimic-negative control (empty plasmid); UTR, untranslated region; RT-qPCR, real-time quantitative polymerase chain reaction; SEM: standard error of the mean.

in the MDA5 signaling pathway was significantly downregulated $(P \leq 0.05)$, and sample-to-positive ratios of virion secretion in the supernatants were elevated at 4 and 5 days after the infection $(P \leq 0.05$; Figures $6 \mathbf{G}, \mathbf{H})$. These data suggest that MDA5 can recognize ALV-J infections and initiate and amplify an innate immune response via the MDA5 signaling pathway in chickens.

\section{MIR-34b-5p Modulates the MDA5 Signaling Pathway and Promotes ALV-J Replication}

As our findings suggested that miR-34b-5p might promote the proliferation and migration of ALV-J-infected DF-1 cells by targeting MDA5, we assessed the effects of miR-34b-5p overexpression on the MDA5 signaling pathway to explore the mechanism by which miR-34b-5p contributes to ALV$J$ infections. In DF-1 cells that overexpressed miR-34b$5 \mathrm{p}$, the mRNA and protein expression level of Env were significantly elevated 3 days after the infection $(P<0.01$; Figures 7A,B). Moreover, ectopic expression of miR-34b-5p significantly inhibited the expression of genes in the MDA5 signaling pathway, including MDA5, IPS-1, IFN $\beta, O A S, M x 1$, and $M H C$ class $I(P \leq 0.05)$, in ALV-J-infected DF-1 cells (Figures 7A,C). The sample-to-positive ratio of virion secreted in the supernatants was also significantly upregulated $(P \leq$ 0.05 ) in DF-1 cells that overexpressed miR-34b-5p during ALV$\mathrm{J}$ infections (Figure 7D). These data indicate that miR-34b-5p targets MDA5 to modulate the MDA5 signaling pathway and further promote ALV-J replication.

\section{DISCUSSION}

We found that the expression of miR-34b-5p was increased in ALV-J-infected tissues and that ectopic expression of miR$34 \mathrm{~b}-5 \mathrm{p}$ accelerated the proliferation and migration of ALVJ-infected cells by targeting MDA5. In vitro experiments revealed that the MDA5 signaling pathway, ALV-J related gene expression, and virion secretion can be regulated by miR$34 \mathrm{~b}-5 \mathrm{p}$. Accumulated evidence has indicated that miRNAs are involved in tumor development, and aberrant miRNA expression is closely associated with tumorigenesis (Zhang et al., 2007; Asangani et al., 2008; Lovat et al., 2011; Tomasetti et al., 2016). To date, several miRNAs, including gga-miR221, gga-miR-222, gga-miR-23b, gga-miR-375, gga-miR-125b, gga-miR-1650, gga-miR-193a, gga-miR-193b, gga-let-7b, gga-let7i, gga-miR-458, gga-miR-1456, gga-miR-1704, gga-miR-1777, gga-miR-1790, and gga-miR-2127, have been reported to be associated with tumorigenesis and the aberrant expression of the retrovirus, ALV-J (Li et al., 2012; Wang et al., 2013a,b; Li H. et al., 2014; Dai et al., 2015; Li et al., 2015). Our previous miRNA sequencing data indicated that miR-34b$5 \mathrm{p}$ expression was increased in ALV-J-infected spleens. We confirmed these results in the present study and discovered that miR-34b-5p also was upregulated in other immune systemrelated tissues.

MiR-34b-5p is a member of the miR-34 family. Previous studies have suggested that members of the miR-34 family may 


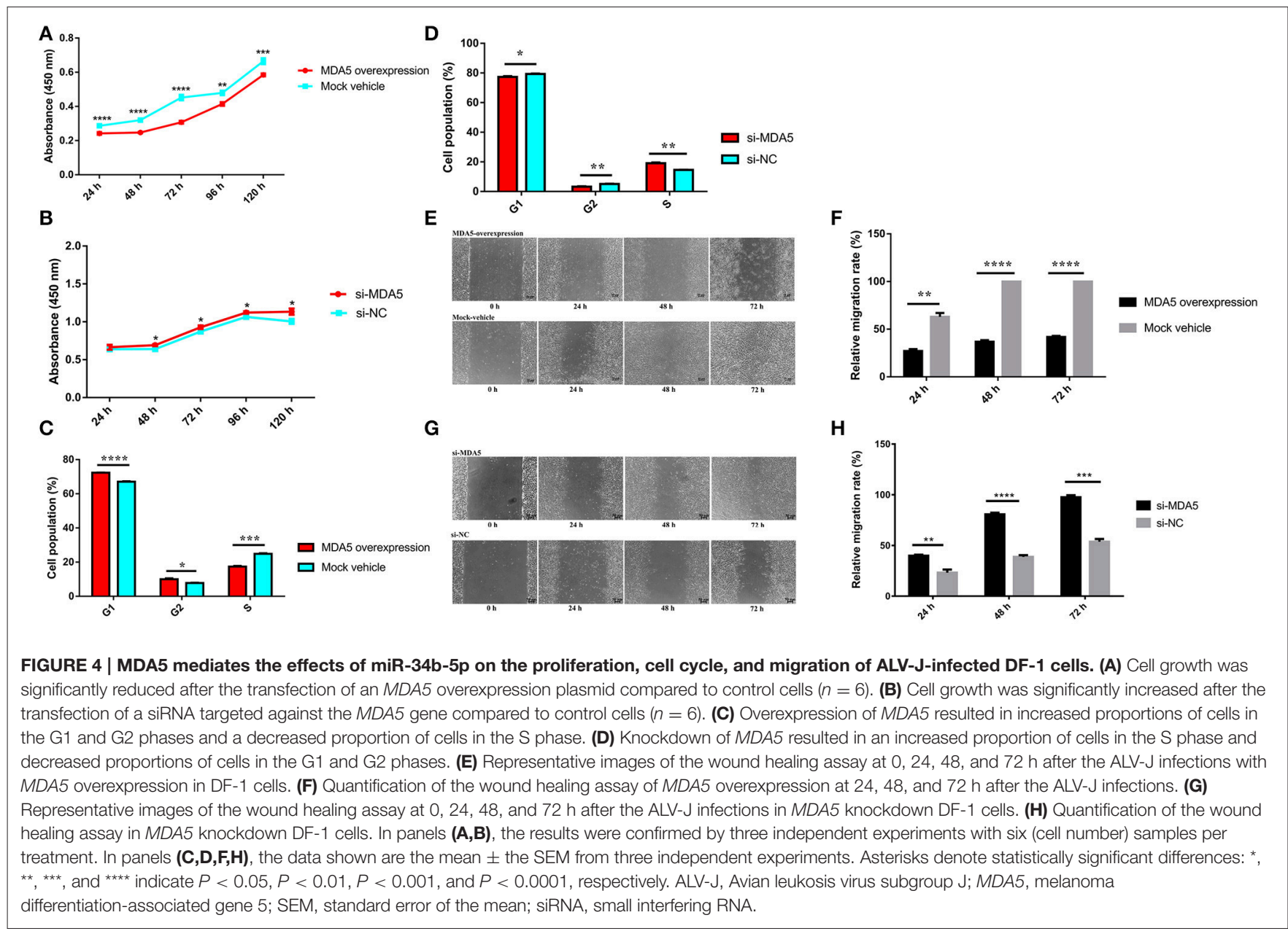

act as tumor suppressors (He et al., 2007; Hermeking, 2010). However, most previous studies have been focused on two other members of this family, miR-34a and miR-34c. It has been welldocumented that miR-34b-5p is involved in the regulation of spermatogenesis (Smorag et al., 2012; Sakurai et al., 2015). In addition, the miR-34b-5p/Notch1 pathway was reported to be important for the oncogenesis involved in minimal deviation adenocarcinoma (Lee H. et al., 2014). To our knowledge, this study is the first study to demonstrate that miR-34b-5p may be involved in the pathogenesis of ALV-J, as it appears to affect many aspects of cancer biology, such as cell proliferationand cell cycle progression. We further investigated the biological functions of miR-34b-5p using an overexpression experiment (Zhao et al., 2015; Shi B. et al., 2016). The functional investigation demonstrated that ectopic expression of miR-34b-5p could accelerate cell proliferation and migration in ALV-J-infected cells. The cell cycle comprises three phases: G1, which involves preparation for DNA synthesis; S, which involves DNA synthesis; and G2, which involves preparation for mitosis (Zhang et al., 2009). We found that miR-34b-5p overexpression increased the proportion of cells in S phase and reduced the proportion of cells in G2 phase, suggesting that it can induce cells from the G1 to the $\mathrm{S}$ phase.
It is well-known that each miRNA can regulate up to hundreds of target genes (Lewis et al., 2005). The use of prediction software revealed that miR-34b-5p may have an effect on a large number of target genes. Among these, three oncogenes, Notch1, c-Met, and CDK6, have been confirmed to be targets of miR-34b-5p (Smorag et al., 2012; Lee C. C. et al., 2014; Li et al., 2016). We predicted that the MDA5 gene was a target of miR-34b-5p using miRDB software and integrative analyses of our previous RNA and small RNA sequencing data (GEO database, accession numbers, GSE63226 and GSE63676, respectively). Subsequently, we confirmed this prediction using RT-qPCR and a dualluciferase reporter assay.

The PRRs of host innate immune system, including Toll-like receptors (TLRs), retinoic acid-inducible gene I-like helicases (RLHs), and nucleotide-oligomerization domain-like receptors (NLRs), can detect viral infections and evoke antiviral responses by producing type I IFNs. MDA5 belongs to the RLH family and it is able to detect viral RNA in the cytoplasm (Pichlmair et al., 2006). The domain architecture of chicken MDA5 comprises two caspase recruitment domains (CARDs), a restriction enzyme domain type 3 (RES III), a helicase conserved C-terminal domain (helicase-C), and a retinoic acid-inducible gene I C-terminal regulatory domain (RIG-I-C-RD) (Lee et al., 2012) During a viral 

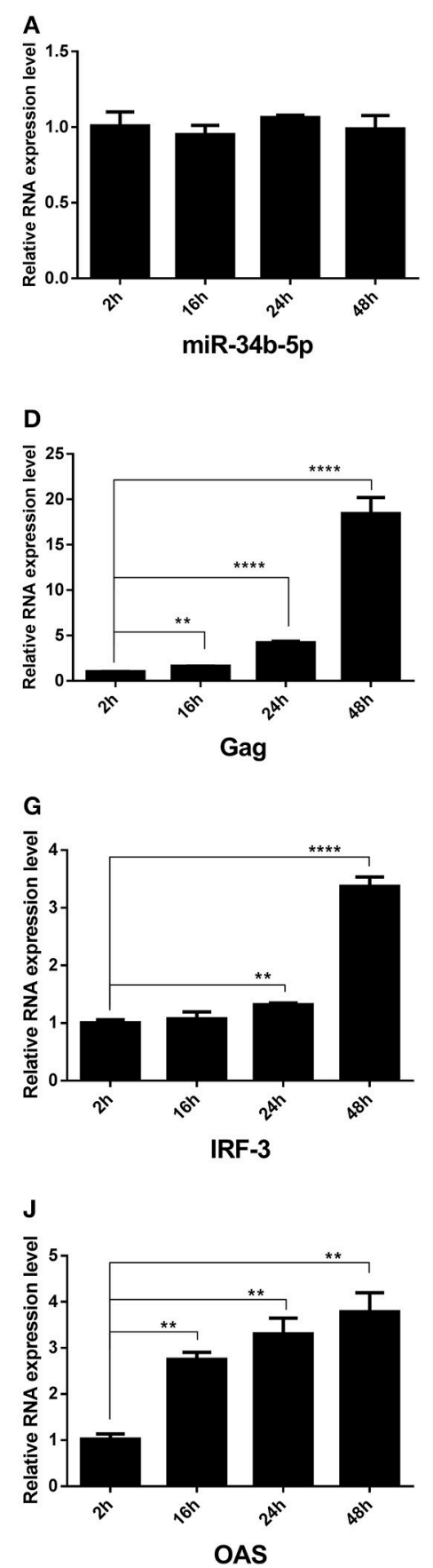
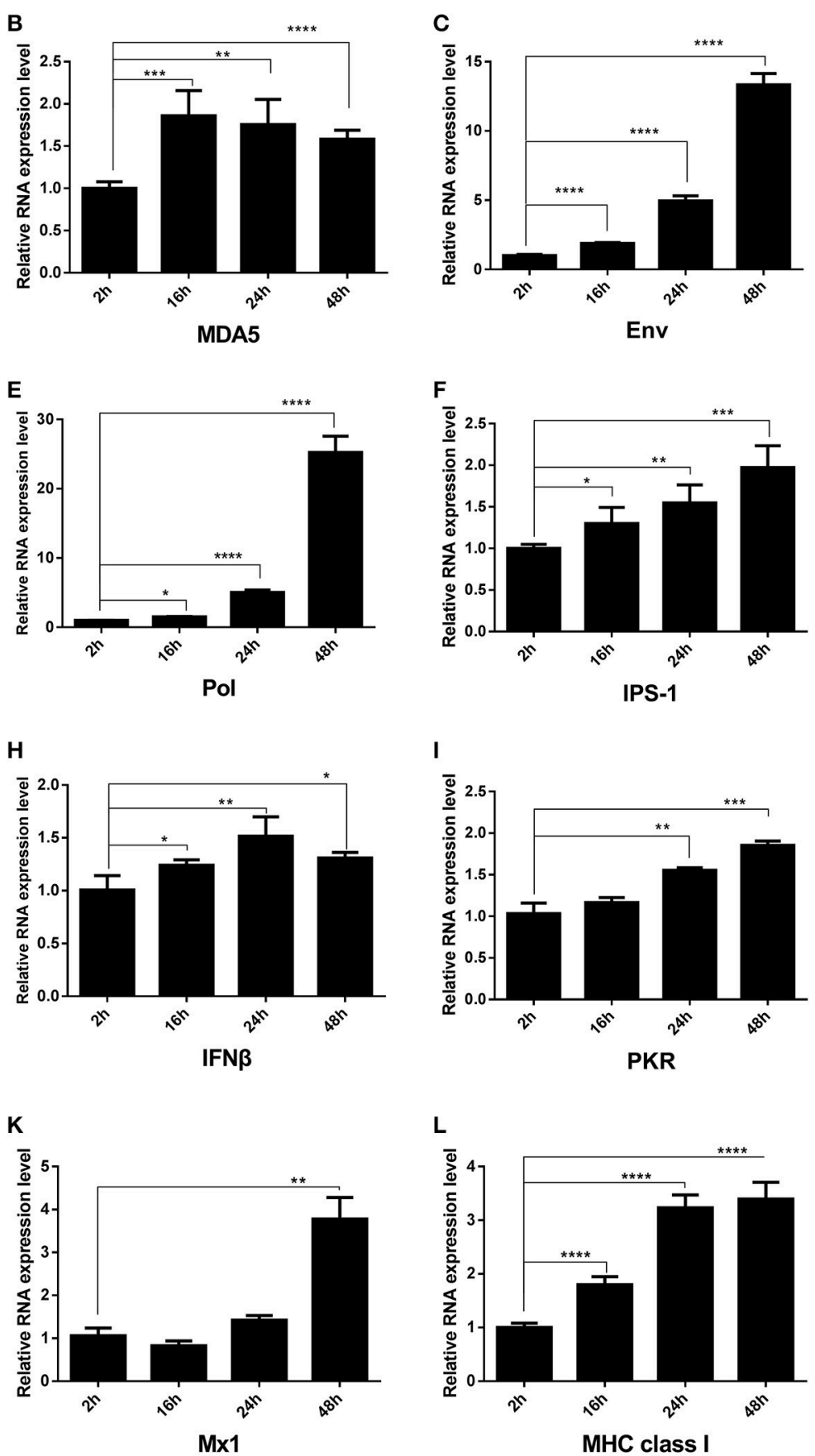

FIGURE 5 | The expression of mRNA of ALV-J env gag, pol and MDA5 signaling pathway-related innate and antiviral genes in chicken DF-1 cells during ALV-J infections. DF-1 cells were infected with ALV-J at TCID50. The change in the expression of (A) miR-34b-5p, (B) MDA5, (C) Env, (D) gag, (E) Pol, (F) IPS-1, (G) IRF-3, (H) IFN $\beta$, (I) PKR, (J) OAS, (K) Mx1, and (L) MHC class I in ALV-J-infected DF-1 cells from 2 to $48 \mathrm{~h}$ after the infection. In all panels, each bar represents the mean \pm the SEM of three independent experiments. Asterisks denote statistically significant differences: ${ }^{*},{ }^{\star *}$, ${ }^{\star \star *}$, and ${ }^{* \star * *}$ indicate $P<0.05, P<0.01$, $P<0.001$, and $P<0.0001$, respectively. ALV-J, Avian leukosis virus subgroup J; IFN $\beta$, interferon- $\beta$; IPS- 1 , interferon- $\beta$ promoter stimulator 1 ; IRF-3, interferon regulatory factor-3; MDA5, melanoma differentiation-associated gene 5; $M H C$, major histocompatibility complex; $M \times 1$, myxovirus (influenza virus) resistance 1 , interferon-inducible protein p78 (mouse); OAS, 2', 5'-oligoadenylate synthetase; PKR, double-stranded RNA-dependent protein kinase; SEM, standard error of the mean; $\mathrm{TCID}_{50}, 50 \%$ tissue culture infectious dose.

infection, MDA5 detects viral RNA that has a helicase domain and transmits a signal via CARDs. The activated CARDs associate with an adaptor protein, IPS-1, and this leads to the recruitment and activation of both canonical and non-canonical inhibitors of NF- $\kappa$ B kinase (IKK) complexes. Furthermore, the activated IKK complexes mediate the phosphorylation of IRF-3, which 

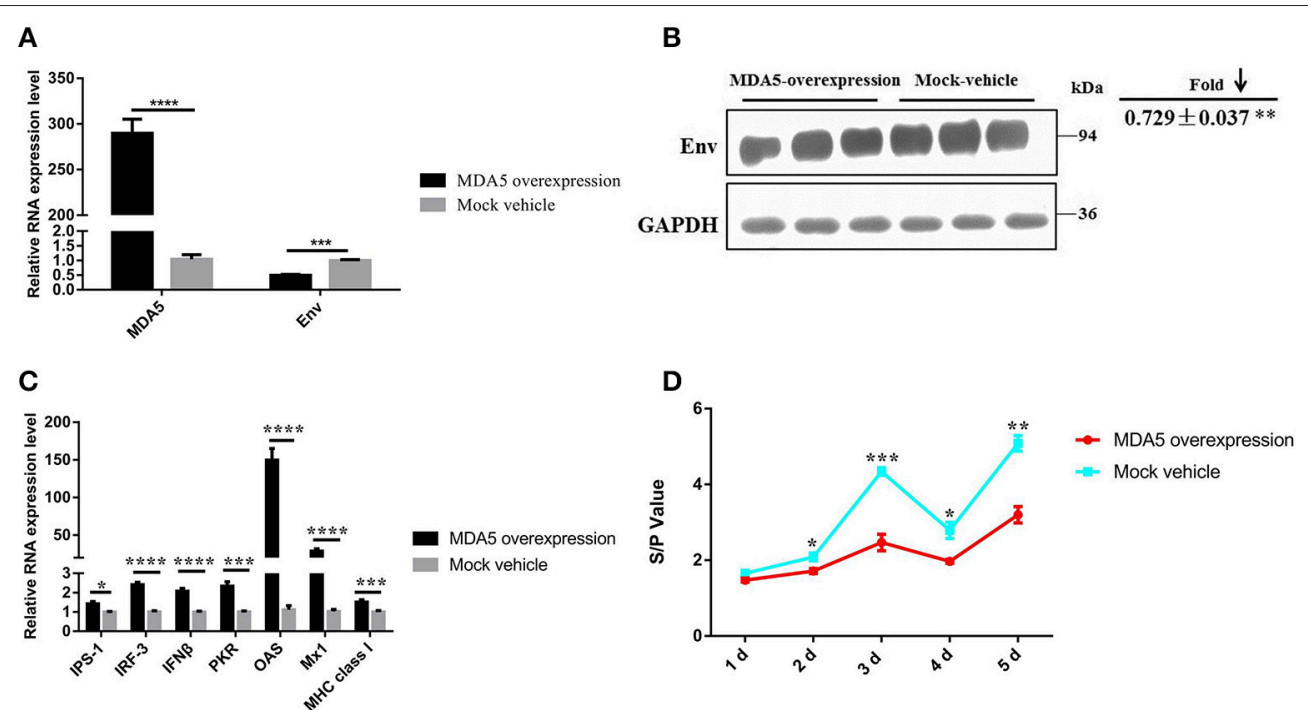

E

$$
\text { F }
$$
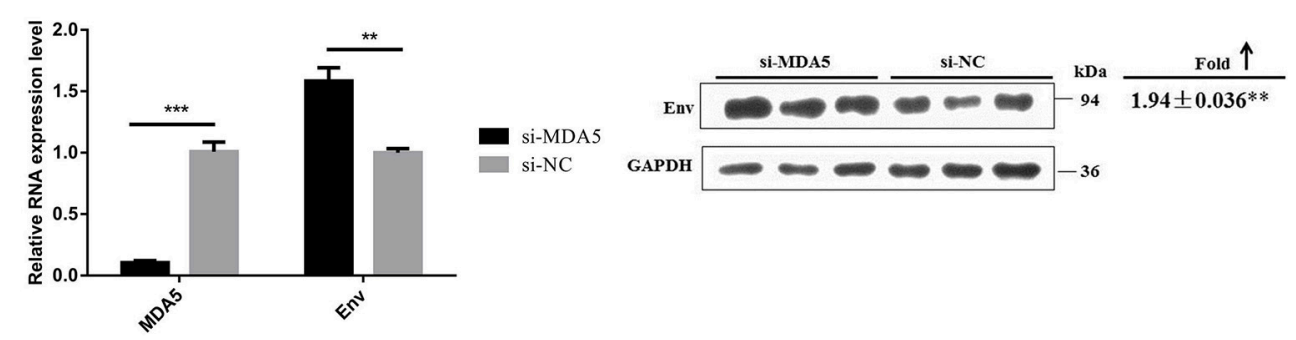

G

H
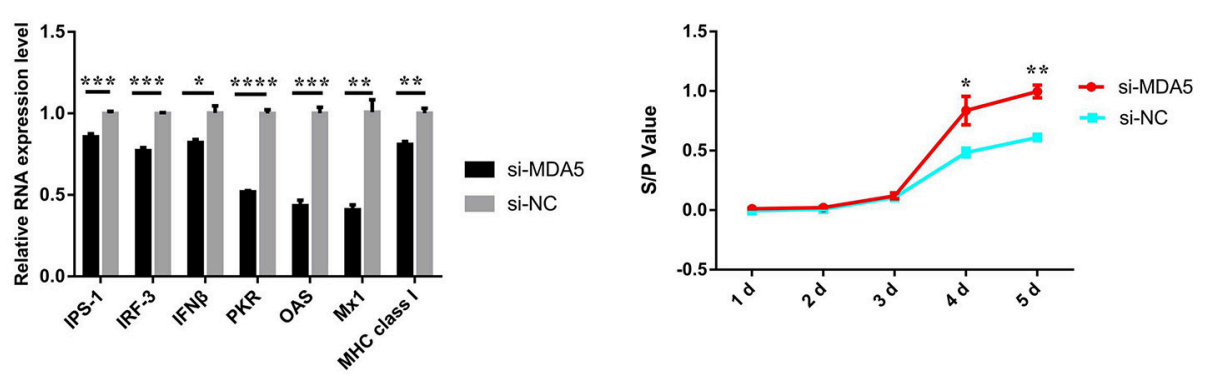

FIGURE 6 | MDA5 can detect ALV-J infections and trigger the MDA5 signaling pathway. The expression of ALV-J env (A) mRNA and (B) protein were downregulated in DF-1 cells that overexpressed MDA5 3 days after the ALV-J infections. (C) The mRNA expression of MDA5 signaling pathway-related innate and antiviral genes was upregulated in DF-1 cells that overexpressed MDA5. (D) The sample-to-positive ratio of virion secreted in the supernatants was downregulated in DF-1 cells that overexpressed MDA5 $(n=6)$. The expression of ALV-J env $(\mathbf{E})$ mRNA and (F) protein were upregulated in MDA5 knockdown DF-1 cells. (G) The mRNA expression of MDA5 signaling pathway-related innate and antiviral genes was downregulated in MDA5 knockdown DF-1 cells. (H) The sample-to-positive ratio of virion secreted in the supernatants was upregulated after MDA5 knockdown by siRNA $(n=6)$. In panels $(\mathbf{A}-\mathbf{C}, \mathbf{E}-\mathbf{G})$, the data shown are the mean \pm the SEM from three independent experiments. In panels $(\mathbf{D}, \mathbf{H})$, the results were confirmed by three independent experiments with six (cell number) samples per treatment. Asterisks denote statistically significant differences: ${ }^{*},{ }^{* \star},{ }^{* \star *}$, and ${ }^{* * * *}$ indicate $P<0.05, P<0.01, P<0.001$, and $P<0.0001$, respectively. ALV-J, Avian leukosis virus subgroup J; MDA5, melanoma differentiation-associated gene 5; SEM, standard error of the mean; siRNA, small interfering RNA.

induces the formation of homodimers and/or heterodimers. The functional IRF dimers translocate to the cell nucleus and interact with IFN-stimulated response elements (ISREs), which leads to the transcription of $I F N \beta$ and a set of IFN-inducible genes ( $P K R$, OAS, Mx1, and MHC class I; Kato et al., 2006; Takeuchi and Akira, 2008; Karpala et al., 2011).

Our results showed that in the natural infection in tissues of broilers caused by ALV-J, the expression level of miR-34b-5p was aberrantly upregulated (Figures 1A,B); whereas, the expression level of its target gene MDA5 was significantly down-regulated (Figures 3A,B). On the other hand, in DF-1 cells infected ALVJ, when miR-34b-5p was normally expressed (Figure 5A), the expression level of MDA5 was up-regulated at $16 \mathrm{~h}, 24 \mathrm{~h}, 48 \mathrm{~h}$ compared with $2 \mathrm{~h}$ after ALV-J infection (Figure 5B). These differences in the expression levels could possibly be due to the subject of time after ALV-J infection between the tissues (spleen) 
A

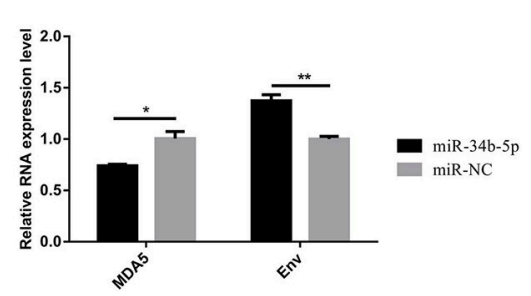

C

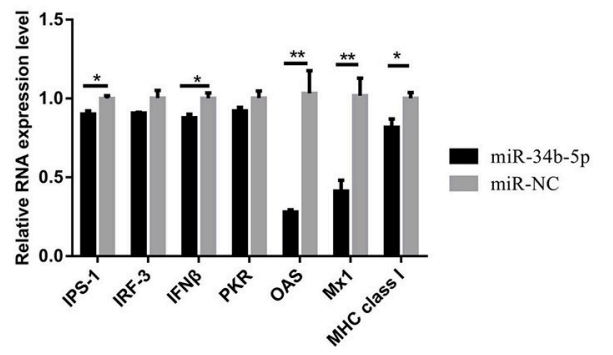

B

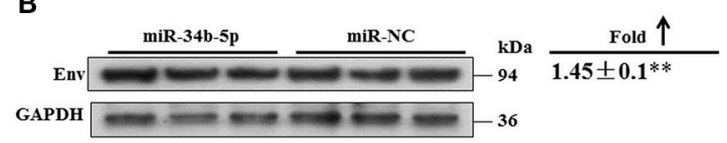

D

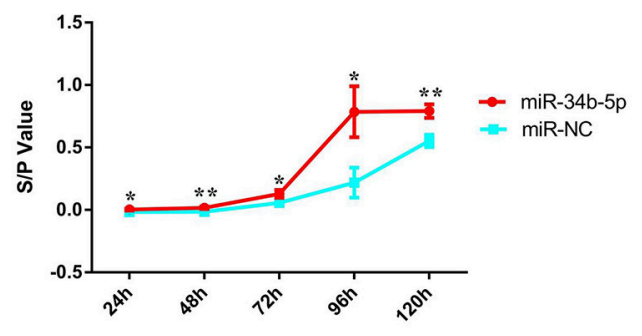

FIGURE 7 | Overexpression of miR-34b-5p suppresses the MDA5 signaling pathway and promotes ALV-J replication. The (A) mRNA and (B) protein expression level of env were upregulated in DF-1 that overexpressed miR-34b-5p cells 3 days after the ALV-J infections. (C) The expression of mRNA of MDA5 signaling pathway-related innate and antiviral genes was downregulated in DF-1 cells that overexpressed miR-34b-5p. (D) The sample-to-positive ratio of virion secreted in supernatants was upregulated in DF-1 cells that overexpressed miR-34b-5p $(n=6)$. In panels (A-C), the values are expressed as the mean \pm the SEM of three independent experiments. In panel (D), the results were confirmed by three independent experiments with six (cell number) samples per treatment. Asterisks denote statistically significant differences: * and ${ }^{* *}$ indicate $P<0.05$ and $P<0.01$, respectively. ALV-J, Avian leukosis virus subgroup J; MDA5, melanoma differentiation-associated gene 5; SEM, standard error of the mean.

and DF-1 cells i.e., the time of spleen-infected broiler was longer (140-day-old spontaneous infectious broilers) than those in DF1 cells infected for not more than $48 \mathrm{~h}$. It has been reported that the ALV-J viral stock could cause fibroblastic sarcoma in chickens within 14 days but gave rise to a moderate transformation in the culture of primary chicken embryonic fibroblast cells (Wang et al., 2015). In order to confirm the assumption above, we carried out miR-34b-5p mimic transfection in DF-1 and then infected with ALV-J, after miR-34b-5p mimic transfection, the RNA expression level of miR-34b-5p was significantly upregulated than that in NC group (Supplementary Figure 1A). In addition, when miR-34b-5p was overexpressed, the expression level of MDA5 was downregulated than that in NC group (Supplementary Figure 1B). These results (ALV-J-infected DF-1 cells with miR-34b-5p overexpression; Supplementary Figure $1 \mathrm{~A}, \mathrm{~B})$ are consistent with natural ALV-J infection broilers (Figures 1, 3A,B). Furthermore, we investigated the mRNA expression level of ALV-J-related genes after miR-34b$5 p$ overexpressed in DF-1 cells; the mRNA expression level of Env (Supplementary Figure 1C), Gag (Supplementary Figure 1D) and Pol (Supplementary Figure 1E) were upregulated in ALVJ-infected DF-1 cells compared with that in NC group at 2$48 \mathrm{~h} \mathrm{ALV-J}$ infection time points. Taken together, these data suggest that miR-34b-5p could inhibit MDA5 to promote ALV-J replication in DF-1 cells.

Moreover, we found that MDA5 plays an important role in controlling the proliferation, cell cycle progression, and migration of ALV-J-infected cells (Figure 4). Recently, antiviral signaling pathways have attracted a great deal of attention from researchers and there are many studies on the cellular pathways involved in ALV-J infections. This research is necessary to develop methods to control viral infections and/or help to devise treatments for the infections. At present, several pathways have been reported to play important roles in ALV-J replication and infection, including the phosphoinositide 3kinase $(\mathrm{PI} 3 \mathrm{~K}) / \mathrm{AK}$ mouse strain thymic lymphoma gene (Akt) pathway, the ERK/ activator protein 1 (AP1) pathway, the MAPK signaling pathway, the wingless-related integration site (Wnt) signaling pathway, cytokine-cytokine receptor interaction, the Janus kinase (JAK)/signal transducers and activators of transcription (STAT) signaling pathway and the RLR signaling pathway (Feng et al., 2011; Wang et al., 2013a; Dai et al., 2016). We observed that MDA5 can affect the MDA5 signaling pathway, and there was a clear correlation between this pathway and virion secretion. These observations strongly indicate that the MDA5 signaling pathway is involved in ALV-J replication. In this study we proposed mechanism of miR-34b-5p actions that mediated MDA5 signaling pathway during ALV-J infections (Figure 8).

In conclusion, our findings revealed that miR-34b-5p was increased in ALV-J-infected tissues and that ectopic expression of miR-34b-5p accelerated the proliferation and migration of ALVJ-infected cells and modulated cell cycle progression by targeting MDA5. Further in vitro investigations revealed that miR-34b-5p can regulate the MDA5 signaling pathway, ALV-J related gene expression, and virion secretion. These results demonstrated that miR-34b-5p targets MDA5 to inhibit the expression of genes in the MDA5 signaling pathway (IPS-1, IRF-3, IFN $\beta$, 


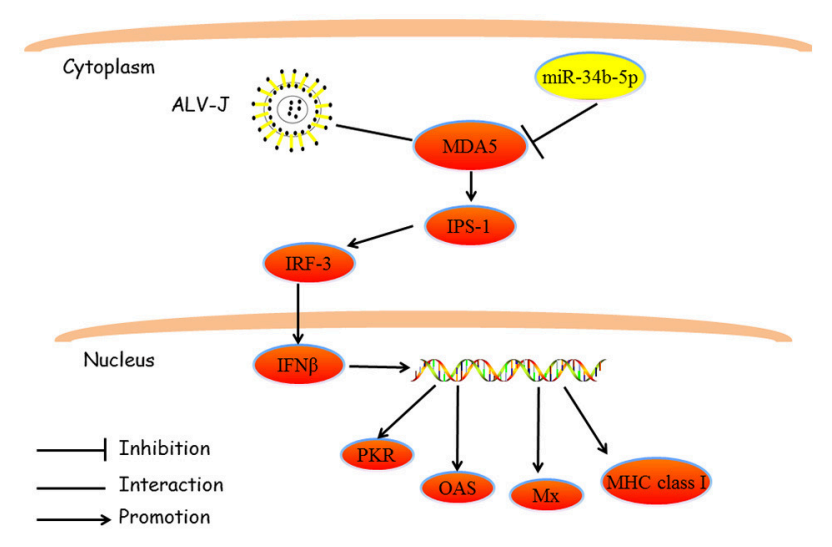

FIGURE 8 | A schematic representation showing the miR-34b-5p-mediated MDA5 signaling pathway during ALV-J infections. This model is based on knowledge of the MDA5-mediated signaling pathway. As a member of the RLH family, MDA5 can detect ALV-J infections in the cytoplasm. Abnormal expression of miR-34b-5p suppressed the function of MDA5, which triggered the signal transduction cascade to induce an IFN $\beta$ response and which, in turn, upregulated downstream antiviral genes (PKR, OAS, Mx1, and MHC class I). MiR-34b-5p inhibited the expression of $M D A 5$, and led to increased proliferation and migration of the ALV-J-infected cells, and promoted ALV-J replication. ALV-J, Avian leukosis virus subgroup J; IFN $\beta$, interferon- $\beta$; MDA5, melanoma differentiation-associated gene 5; $M H C$, major histocompatibility complex; $M \times 1$, myxovirus (influenza virus) resistance 1, interferon-inducible protein p78 (mouse); OAS, 2', 5'-oligoadenylate synthetase; PKR, double-stranded RNA-dependent protein kinase; RLH, retinoic acid-inducible gene I-like helicases.

PKR, OAS, Mx1, and MHC class I), accelerate the proliferation and migration of ALV-J-infected cells and promote ALV-J replication (Figure 8). These findings provide a solid foundation for the understanding of the underlying mechanisms of ALV-

\section{REFERENCES}

Asangani, I. A., Rasheed, S. A., Nikolova, D. A., Leupold, J. H., Colburn, N. H., Post, S., et al. (2008). MicroRNA-21 (miR-21) post-transcriptionally downregulates tumor suppressor Pdcd 4 and stimulates invasion, intravasation and metastasis in colorectal cancer. Oncogene 27, 2128-2136. doi: 10.1038/sj.onc.1210856

Chai, N., and Bates, P. (2006). Na+/H+ exchanger type 1 is a receptor for pathogenic subgroup J avian leukosis virus. Proc. Natl. Acad. Sci. U.S.A. 103, 5531-5536. doi: 10.1073/pnas.0509785103

Dai, M. M., Feng, M., Ye, Y., Wu, X. C., Liu, D., Liao, M., et al. (2016). Exogenous avian leukosis virus-induced activation of the ERK/AP1 pathway is required for virus replication and correlates with virus-induced tumorigenesis. Sci. Rep. 6:19226. doi: 10.1038/srep19226

Dai, Z. K., Ji, J., Yan, Y. M., Lin, W. C., Li, H. X., Chen, F., et al. (2015). Role of gga-miR-221 and gga-miR-222 during tumour formation in chickens infected by subgroup J avian leukosis virus. Viruses 7, 6538-6551. doi: 10.3390/v7122956

Datta, J., Smith, A., Lang, J. C., Islam, M., Dutt, D., Teknos, T. N., et al. (2012). MicroRNA-107 functions as a candidate tumor-suppressor gene in head and neck squamous cell carcinoma by downregulation of protein kinase Cvarepsilon. Oncogene 31, 4045-4053. doi: 10.1038/onc.2011.565

Feng, M., Dai, M. M., Xie, T. T., Li, Z., Shi, M., and Zhang, X. (2016). Innate immune responses in ALV-J infected chicks and chickens with hemangioma in vivo. Front Microbiol. 7:786. doi: 10.3389/fmicb.2016.00786
J pathogenesis and they will contribute to the development of studies on other retroviruses.

\section{AUTHOR CONTRIBUTIONS}

ZL performed the research, analyzed the data, and wrote the paper. QL participated in the study design, analyzed the data, and wrote the paper. HX analyzed the data and wrote the paper. MZ analyzed the data. BA reviewed the manuscript. QN conceived of the study and participated in its design and coordination. $\mathrm{XQZ}$ participated in the design of the study. MF, BC, and XCZ contributed reagents and other resources.

\section{FUNDING}

This research was supported by the Program for New Century Excellent Talents in University (NCET-13-0803), the Natural Scientific Foundation of China (31571269), the Foundation for High-level Talents in Higher Education in Guangdong, China, and the Science and Technology Research Project of Guangzhou (201508020075).

\section{ACKNOWLEDGMENTS}

We thank Assoc. Prof. Weisheng Cao (South China Agricultural University, China) for kindly providing the ALV-J strain, SCAUHN06, used in this study. We would also like to thank Prof. Aijian Qin (Yangzhou University, China) for kindly providing the ALV-J-specific monoclonal antibody, JE-9.

\section{SUPPLEMENTARY MATERIAL}

The Supplementary Material for this article can be found online at: http://journal.frontiersin.org/article/10.3389/fcimb. 2017.00017/full\#supplementary-material

Feng, S., Cong, S., Zhang, X., Bao, X., Wang, W., Li, H., et al. (2011). MicroRNA-192 targeting retinoblastoma 1 inhibits cell proliferation and induces cell apoptosis in lung cancer cells. Nucleic Acids Res. 39, 6669-6678. doi: 10.1093/nar/gkr232

He, L., He, X., Lim, L. P., de Stanchina, E., Xuan, Z., Liang, Y., et al. (2007). A microRNA component of the p53 tumour suppressor network. Nature 447, 1130-1134. doi: 10.1038/nature05939

Hermeking, H. (2010). The miR-34 family in cancer and apoptosis. Cell Death Differ. 17, 193-199. doi: 10.1038/cdd.2009.56

Karpala, A. J., Stewart, C., McKay, J., Lowenthal, J. W., and Bean, A. G. (2011). Characterization of chicken Mda5 activity: regulation of IFNbeta in the absence of RIG-I functionality. J. Immunol. 186, 5397-5405. doi: 10.4049/jimmunol.1003712

Kato, H., Takeuchi, O., Sato, S., Yoneyama, M., Yamamoto, M., Matsui, K., et al. (2006). Differential roles of MDA5 and RIG-I helicases in the recognition of RNA viruses. Nature 441, 101-105. doi: 10.1038/nature04734

Ke, S. H., and Madison, E. L. (1997). Rapid and efficient site-directed mutagenesis by single-tube "megaprimer" PCR method. Nucleic Acids Res. 25, 3371-3372. doi: $10.1093 /$ nar/25.16.3371

Lee, C. C., Wu, C. C., and Lin, T. L. (2012). Characterization of chicken melanoma differentiation-associated gene 5 (MDA5) from alternative translation initiation. Comp. Immunol. Microbiol. Infect. Dis. 35, 335-343. doi: 10.1016/j.cimid.2012.02.004 
Lee, C. C., Wu, C. C., and Lin, T. L. (2014). Chicken melanoma differentiationassociated gene 5 (MDA5) recognizes infectious bursal disease virus infection and triggers MDA5-related innate immunity. Arch. Virol. 159, 1671-1686. doi: 10.1007/s00705-014-1983-9

Lee, H., Kim, K. R., Cho, N. H., Hong, S. R., Jeong, H., Kwon, S. Y., et al. (2014). MicroRNA expression profiling and Notch1 and Notch2 expression in minimal deviation adenocarcinoma of uterine cervix. World J. Surg. Oncol. 12:334. doi: 10.1186/1477-7819-12-334

Lewis, B. P., Burge, C. B., and Bartel, D. P. (2005). Conserved seed pairing, often flanked by adenosines, indicates that thousands of human genes are microRNA targets. Cell 120, 15-20. doi: 10.1016/j.cell.2004.12.035

Li, H., Ji, J., Xie, Q., Shang, H., Zhang, H., Xin, X., et al. (2012). Aberrant expression of liver microRNA in chickens infected with subgroup J avian leukosis virus. Virus Res. 169, 268-271. doi: 10.1016/j.virusres.2012.07.003

Li, H., Shang, H., Shu, D., Zhang, H., Ji, J., Sun, B., et al. (2014). Gga-miR-375 plays a key role in tumorigenesis post subgroup J avian leukosis virus infection. PLoS ONE 9:e90878. doi: 10.1371/journal.pone.0090878

Li, T., Yan, X., Jiang, M., and Xiang, L. (2016). The comparison of microRNA profile of the dermis between the young and elderly. J. Dermatol. Sci. 82, 75-83. doi: 10.1016/j.jdermsci.2016.01.005

Li, Y., Liu, X., Yang, Z., Xu, C., Liu, D., Qin, J., et al. (2014). The MYC, TERT, and ZIC1 genes are common targets of viral integration and transcriptional deregulation in avian leukosis virus subgroup J-induced myeloid leukosis. $J$ Virol. 88, 3182-3191. doi: 10.1128/JVI.02995-13

Li, Z., Chen, B., Feng, M., Ouyang, H., Zheng, M., Ye, Q., et al. (2015). MicroRNA23b promotes avian leukosis virus subgroup J (ALV-J) replication by targeting IRF1. Sci. Rep. 5:10294. doi: 10.1038/srep10294

Livak, K. J., and Schmittgen, T. D. (2001). Analysis of relative gene expression data using real-time quantitative PCR and the 2(-delta delta C(T)) method. Methods 25, 402-408. doi: 10.1006/meth.2001.1262

Lovat, F., Valeri, N., and Croce, C. M. (2011). MicroRNAs in the pathogenesis of cancer. Semin. Oncol. 38, 724-733. doi: 10.1053/j.seminoncol.2011. 08.006

Pan, W., Gao, Y., Qin, L., Ni, W., Liu, Z., Yun, B., et al. (2012). Genetic diversity and phylogenetic analysis of glycoprotein GP85 of ALVJ isolates from mainland China between 1999 and 2010: coexistence of two extremely different subgroups in layers. Vet. Microbiol. 156, 205-212. doi: 10.1016/j.vetmic.2011.10.019

Payne, L. N. (1998). Retrovirus-induced disease in poultry. Poult. Sci. 77, 1204-1212. doi: 10.1093/ps/77.8.1204

Payne, L. N., and Nair, V. (2012). The long view: 40 years of avian leukosis research. Avian. Pathol. 41, 11-19. doi: 10.1080/03079457.2011.646237

Pichlmair, A., Schulz, O., Tan, C. P., Naslund, T. I., Liljestrom, P., Weber, F., et al. (2006). RIG-I-mediated antiviral responses to single-stranded RNA bearing 5'-phosphates. Science 314, 997-1001. doi: 10.1126/science.1132998

Reed, L. J., and Muench, H. (1938). A simple method of estimating fifty percent endpoints. Am. J. Hyg. 27, 493-497.

Sakurai, K., Mikamoto, K., Shirai, M., Iguchi, T., Ito, K., Takasaki, W., et al. (2015). MicroRNA profiling in ethylene glycol monomethyl ether-induced monkey testicular toxicity model. J. Toxicol. Sci. 40, 375-382. doi: 10.2131/jts. 40.375

Shi, B., Gao, H., Zhang, T., and Cui, Q. (2016). Analysis of plasma microRNA expression profiles revealed different cancer susceptibility in healthy young adult smokers and middle-aged smokers. Oncotarget 7, 21676-21685. doi: 10.18632/oncotarget.7866

Shi, W., Bruce, J., Lee, M., Yue, S., Rowe, M., Pintilie, M., et al. (2016). MiR449a promotes breast cancer progression by targeting CRIP2. Oncotarget 7, 18906-18918. doi: 10.18632/oncotarget.7753

Smorag, L., Zheng, Y., Nolte, J., Zechner, U., Engel, W., and Pantakani, D. V. (2012). MicroRNA signature in various cell types of mouse spermatogenesis: evidence for stage-specifically expressed miRNA-221, -203 and $-34 \mathrm{~b}-5 \mathrm{p}$ mediated spermatogenesis regulation. Biol. Cell. 104, 677-692. doi: 10.1111/boc.201200014

Stedman, N. L., and Brown, T. P. (1999). Body weight suppression in chickens naturally infected with avian leukosis virus subgroup J. Avian Dis. 43, 604-610. doi: $10.2307 / 1592664$

Sun, W., Julie, L. Y., Huang, H. D., Shyy, J. Y., and Chien, S. (2010). MicroRNA: a master regulator of cellular processes for bioengineering systems. Annu. Rev. Biomed. Eng. 12, 1-27. doi: 10.1146/annurev-bioeng-070909-105314

Takeuchi, O., and Akira, S. (2008). MDA5/RIG-I and virus recognition. Curr. Opin. Immunol. 20, 17-22. doi: 10.1016/j.coi.2008.01.002

Tomasetti, M., Amati, M., Santarelli, L., and Neuzil, J. (2016). MicroRNA in metabolic re-programming and their role in tumorigenesis. Int. J. Mol. Sci. 17:754. doi: 10.3390/ijms17050754

Tsai, K. W., Wu, C. W., Hu, L. Y., Li, S. C., Liao, Y. L., Lai, C. H., et al. (2011). Epigenetic regulation of miR-34b and miR-129 expression in gastric cancer. Int. J. Cancer 129, 2600-2610. doi: 10.1002/ijc.25919

Wang, Q., Gao, Y., Ji, X., Qi, X., Qin, L., Gao, H., et al. (2013a). Differential expression of microRNAs in avian leukosis virus subgroup J-induced tumors. Vet. Microbiol. 162, 232-238. doi: 10.1016/j.vetmic.2012.10.023

Wang, Q., Ji, X. L., Gao, Y. L., Qi, X. L., Wang, X. J., Wang, Y. Q., et al. (2013b). Overexpression of microRNA gga-miR-1650 decreases the replication of avian leukosis virus subgroup J in infected cells. J. Gen. Virol. 94, 2287-2296. doi: 10.1099/vir.0.054007-0

Wang, Y., Xu, S., Li, S., Su, H., Chang, S., Li, Y., et al. (2015). Lamivudine inhibits the replication of ALV-J associated acutely transforming virus and its helper virus and tumor growth in vitro and in vivo. Front. Microbiol. 6:1306. doi: $10.3389 /$ fmicb.2015.01306

Wong, N., and Wang, X. (2015). MiRDB: an online resource for microRNA target prediction and functional annotations. Nucleic Acids Res. 43, D146-D152. doi: $10.1093 /$ nar/gku1104

Zeng, X., Liu, L., Hao, R., and Han, C. (2014). Detection and molecular characterization of J subgroup avian leukosis virus in wild ducks in China. PLoS ONE 9:e94980. doi: 10.1371/journal.pone.0094980

Zhang, M., Xie, R., Hou, W., Wang, B. L., Shen, R., Wang, X. M., et al. (2009). PTHrP prevents chondrocyte premature hypertrophy by inducing cyclin-D1dependent Runx2 and Runx3 phosphorylation, ubiquitylation and proteasomal degradation. J. Cell Sci. 122, 1382-1389. doi: 10.1242/jcs.040709

Zhang, W., Dahlberg, J., and Tam, W. (2007). MicroRNAs in tumorigenesis: a primer. Am. J. Pathol. 171, 728-738. doi: 10.2353/ajpath.2007.070070

Zhao, J. J., Yang, J. H., Lin, J. H., Yao, N., Zhu, Y. H., Zheng, J. L., et al. (2009). Identification of miRNAs associated with tumorigenesis of retinoblastoma by miRNA microarray analysis. Childs Nerv. Syst. 25, 13-20. doi: 10.1007/s00381-008-0701-X

Zhao, L. Y., Yao, Y., Han, J., Yang, J., Wang, X. F., Tong, D. D., et al. (2014). MiR638 suppresses cell proliferation in gastric cancer by targeting Sp2. Dig. Dis. Sci. 59, 1743-1753. doi: 10.1007/s10620-014-3087-5

Zhao, X. M., Liu, K. Q., Zhu, G., He, F., Duval, B., Richer, J. M., et al. (2015). Identifying cancer-related microRNAs based on gene expression data. Bioinformatics 31, 1226-1234. doi: 10.1093/bioinformatics/btu811

Conflict of Interest Statement: The authors declare that the research was conducted in the absence of any commercial or financial relationships that could be construed as a potential conflict of interest.

Copyright (c) 2017 Li, Luo, Xu, Zheng, Abdalla, Feng, Cai, Zhang, Nie and Zhang This is an open-access article distributed under the terms of the Creative Commons Attribution License (CC BY). The use, distribution or reproduction in other forums is permitted, provided the original author(s) or licensor are credited and that the original publication in this journal is cited, in accordance with accepted academic practice. No use, distribution or reproduction is permitted which does not comply with these terms. 